\title{
S s.man \\ Older Patients' Experience of Living With Cognitive Impairment Related to Hormone Therapy for Breast Cancer
}

\section{Yasuko Tenda}

Hiroshima University: Hiroshima Daigaku

Mika Miyashita ( $\sim$ mika-tky@umin.ac.jp)

Hiroshima University https://orcid.org/0000-0002-0569-6487

\section{Akimitsu Yamada}

Yokohama City University: Yokohama Shiritsu Daigaku

\section{Chikako Shimizu}

National Center for Global Health and Medicine: Kokuritsu Kenkyu Kaihatsu Hojin Kokuritsu Kokusai Iryo Kenkyu Center

\section{Kanako Nakayama}

National Center for Global Health and Medicine: Kokuritsu Kenkyu Kaihatsu Hojin Kokuritsu Kokusai Iryo Kenkyu Center

\section{Naoko Honma}

Toho University Faculty of Medicine Graduate School of Medicine: Toho Daigaku Igakubu Daigakuin Igaku Kenkyuka

\section{Naruto Taira}

Okayama University: Okayama Daigaku

\section{Masataka Sawaki}

Aichi Cancer Center: Aichi-ken Gan Center

\section{Research Article}

Keywords: Breast cancer, Hormone therapy, Older women, Cognitive impairment, Comprehensive geriatric assessment

Posted Date: May 7th, 2021

DOI: https://doi.org/10.21203/rs.3.rs-413974/v1

License: (c) (1) This work is licensed under a Creative Commons Attribution 4.0 International License.

Read Full License 


\section{Abstract \\ Purpose}

Patients with breast cancer may develop cognitive impairment as a result of hormone therapy and aging. This study aimed to describe older patients' experience of cognitive impairment related to hormone therapy for breast cancer.

\section{Methods}

A qualitative and descriptive design was used. Semi-structured interviews were conducted for 11 patients to ask about their experience in daily life related to their cognitive impairment. Study participants were evaluated by cancer-specific geriatric assessment. Inductive content analysis was conducted using interview records.

\section{Results}

Three themes emerged from the patients' narrative data: recognizing of cognitive impairments, impacts on mental health, and coping with cognitive impairments. Recognizing of cognitive function consisted of two categories: perception of cognitive changes and acknowledging their cognitive function status through interacting with others. Impacts on mental health consisted of six categories: decreased motivation for social activities, upset by perception of cognitive decline, concerns about impacts of cognitive impairment on treatment of breast cancer, concerns about care burden of their family, concerns about progressing of cognitive impairments, and feeling secure that their cognitive impairment is not unusual. Coping with cognitive impairments consisted of two categories: coping with problems related to cognitive impairment and trying to maintain and improve cognitive function.

\section{Conclusion}

Medical professionals should perform a comprehensive evaluation of older patients receiving hormone therapy for breast cancer.

\section{Introduction}

Managing the adverse events of cancer treatment is an important issue. Cognitive problems can be caused by treatments other than chemotherapy, such as hormone therapy, and other factors, such as age and genetic polymorphism, may increase the risk [1]. Cognitive impairment is a decline in cognitive function that is a multidimensional concept, which includes a decline in attention, concentration, executive function, information processing speed, language, visuospatial ability, psychomotor ability, 
learning, and memory [2]. Although the severity of cancer therapy-related cognitive impairment is minor, it can adversely affect quality of life (QOL) $[3,4]$.

The main treatments for breast cancer are surgery, radiation therapy, and drug therapy, and it is common to combine these treatments. Approximately $80 \%$ of Japanese breast cancer patients are hormone receptor positive [5]. Older people have a higher rate of being hormone receptor positive [6]. Aromatase inhibitors that are administered to older patients with breast cancer exert a therapeutic effect by lowering estrogen levels in the body in order to reduce the supply and stimulation of hormones and suppress the growth of cancer cells. In addition, selective estrogen receptor modulators, which can block the effects of estrogen on breast cancer, are administered to older breast cancer patients. Estradiol, an estrogen hormone, may have neuroprotective properties in various experimental models of traumatic brain injury and neurodegenerative diseases [7]. Given the widespread presence of estradiol throughout the brain and its neuroprotective properties, a reduction in estrogen levels in the body due to hormone therapy may contribute to cognitive decline [7].

Cognitive dysfunction in the elderly lowers QOL and adversely affects daily life [8]. Older people are at risk of developing cancer-related cognitive impairment [9]. However, few studies have focused on cognitive dysfunction in older patients receiving hormone therapy for breast cancer. A review paper suggested that hormone therapy may affect a wide range of cognitive functions in young breast cancer patients such as learning, memory, processing speed, language, and executive function [10]. Quantitative studies of older breast cancer patients who self-reported cognitive impairment due to hormone therapy did not reveal cognitive impairment by neuropsychological examination [11]. Older breast cancer patients receiving hormone therapy are more likely to experience changes in cognitive function from both age and hormone therapy; thus, it is difficult to grasp the actual state of cognitive dysfunction in older breast cancer patients by quantitative research alone. Older people may have a general health problem with multiple causes called geriatric syndrome. A previous study reported that comprehensive geriatric assessment could identify geriatric syndrome [12] and identify latent problems that are related to cancer therapy [13].

The aim of this study was to describe older patients' experience of living with cognitive impairment related to hormone therapy for breast cancer.

\section{Methods}

This study used a qualitative, descriptive, and cross-sectional design to explore older patients' experience of cognitive impairment related to hormone therapy for breast cancer.

\section{Participants and recruitment}

Eligible patients were women aged between 70 and 85 years old who had undergone surgery for breast cancer, were receiving adjuvant hormone therapy, had a Mini-Mental State Examination (MMSE) [14] score of 24 or higher, had been informed of their diagnosis, were able to speak and write in Japanese, consented to record interview data with an IC recorder, and consented to participate in this study. 
Exclusion criteria were women with breast cancer who had received whole brain irradiation or chemotherapy, had been diagnosed with dementia, had a history of traumatic brain injury or cerebrovascular disease, had recurrence or distant metastasis of breast cancer, had psychiatric problems, had been diagnosed with psychiatric disease, or had been judged to be unable to participate in this study by the researcher due to physical or psychiatric problems.

\section{Procedure}

Participants received a leaflet for recruiting study participants from the physician of a breast clinic in Hiroshima Prefecture. The patients were tested with the MMSE. If the patient's MMSE score was 24 or higher, the first author explained the study aim. The patients who understood and agreed to participate in the study signed a consent form.

\section{Data collection}

The first author explained cancer therapy-related cognitive impairment and its symptoms to the participants. Then, a semi-structured interview was conducted to explore the participants' experience of cognitive impairments in daily life after receiving hormone therapy. The interview included the following questions: (1) What symptoms of cognitive impairment did you experience after receiving hormone therapy? (2) How did you feel when you perceived that you had cognitive impairment? (3) How is your relationship with your significant other? The length of the interviews was from 18 minutes to 83 minutes (median $=41$ minutes).

The cancer-specific geriatric assessment (CSGA), which is a scale for comprehensive geriatric assessment for cancer treatment patients, was used. The CSGA performs a risk assessment of complications in older cancer patients and the required medical assistance to increase their physical function reserve [15]. This scale is constructed from demographic information (age, sex, marital status, family, job, and educational history) and six indicators: physical function (Barthel index, instrumental activities of daily living, Karnofsky performance rating scale, and number of falls in last 6 months), mental health (mental health inventory-17 [MHI-17]) [16], social function (medical outcomes study [MOS] social activity limitations measure), social support (medical outcomes study social support survey [MOS SSS])[17], and nutrition (body mass index [BMI], percent unintentional weight loss in the last 6 months). The range of the mean score of each subscale (anxiety, depression, behavioral control, and positive affect) of the MHI-17 was 1.0 to 6.0. The scores of the MOS SSS subscales (emotional/informational, tangible, affectionate, and positive social interaction) and total score were converted by 100 . Higher scores indicate better status in both the MHI-17 and the MOS SSS. In addition, the data of medical variables were provided by the physician.

\section{Data analysis}

Inductive content analysis was conducted using the interview records. This analysis included three steps: open coding, creating categories, and abstraction [18]. The first author developed codes using the 
research question "what is the experience of older women with cognitive impairment related to hormone therapy for breast cancer?". The second author reviewed the codes and developed final codes. Subcategories were generated from codes with similar meanings, and were grouped according to similarity or dissimilarity. Finally, themes were generated through the abstraction process.

Descriptive statistics of CSGA Japanese version, demographic variables, and medical variables were calculated.

\section{Results}

\section{Characteristics of study participants}

Thirty-one candidate patients were recruited by the physician of the clinic. Eleven patients who had symptoms of cognitive impairment agreed to participate in this study. Table 1 shows the study participants' characteristics. All patients had an MMSE score of 27 or over. The age range of the study participants was 70-81 (mean age $=74$ ) years old. The CSGA scores of the study participants are shown in Table 2. All patients achieved a perfect score on the Barthel Index. One patient (Participant 9) had an MHI-17 score of less than 3 points, one patient (Participant 1) had a low MOS Social Activity Limitations Measure score, and one patient (Participant 5) had a MOS SSS total score of less than 40 points.

\section{Experiences of cognitive impairment}

\section{Theme 1: Recognizing of cognitive function}

\section{Perception of cognitive changes}

All patients perceived symptoms of cognitive changes. Patients recognized their decline of memory function, difficulties of expressing themselves in words, decreased efficiency, deficit of concentration, decline of ability to understand, and awareness of confusion.

Eight patients had difficulties remembering things that were not part of their routine, previous thoughts, or responsibilities. Three patients forgot that they had bought goods or taken medicine. In addition, the patients could not recall what they were trying to do or what they had said. One patient described the experience as follows:

I went to pick up some things and wondered what I had come for. When I went back to where I was, I realized what it was and I went to get it. I had to go there twice (Participant 10).

All ten patients reported difficulties expressing what they wanted to say. Four patients reported that they were less efficient than before when doing the housework and tidying up.

I'm worried that I was disorganized. When I cook, I go back and forth, but I'm not making progress.... Especially in the case of cooking... I sometimes wonder what things are used for. I took out soup stock 
and remembered that, oh yeah, I was thinking of making miso soup. It has been like this a lot lately. (Participant 9).

In addition, seven patients experienced problems of concentration when listening to others speak and performing their daily responsibilities. Two patients described difficulties understanding TV, books, and documents. One patient felt that their brain function was unusually confused:

Somehow, it feels like the blood in my brain makes a noise. Something is wrong... I wonder if my head got messed up (Participant 8).

On the other hand, there was one patient who acknowledged that they felt their cognitive function improved. One patient said as follows:

Recently, words come out right away. I often go out and talk to others (Participant 11).

\section{Acknowledging their cognitive function status through interacting with others}

Nine patients perceived their cognitive function status by comparing their cognitive function with the cognitive functions of people of the same age. Three patients felt their cognitive function was poor compared to their husbands:

My daughter told me that she had already said something. Eh? I didn't remember hearing it. My husband isn't like that (Participant 1).

Two patients described the level of their cognitive function as equal to or better than others:

Especially when people's names don't come out easily. The other person is the same. Ha ha ha... The other person couldn't remember my name either(Participant 3).

In addition, three patients recognized their cognitive function by comments from their significant others. One patient described her experience as follows:

My husband laughed and told me that it was the beginning of cognitive impairment (Participant 3).

\section{Theme 2: Impacts on mental health}

\section{Decreased motivation for social activities}

Four participants lost interest in and motivation for activities, such as watching television or reading newspapers, and social activities such as their hobbies. Some patients reported that they could no longer read newspapers as easily as they used to, found it difficult to enjoy watching television, or wanted to stop learning. One patient said the following:

I feel like my motivation has diminished. Hobbies or anything. I feel that the desire to do that and the desire to do this has disappeared. The lessons last only one year. Ha ha ha. I try to do exercises or 
activities like that, but I suddenly want to quit on the way. So I can't continue to do them. (Participant 1).

Two patients mentioned that they were less likely to go out. Another patient told a researcher that she felt withdrawn at home and did not like to be invited to events by others.

My husband goes out to take pictures with his camera. My husband invited me to go, but I said, "I won't go. Please go alone" (Participant 6).

\section{Upset by perception of cognitive decline}

Two patients suffered distress by noticing that their cognition was not what it used to be. Five patients stated that they were shocked at their behavior and felt that their cognitive function had declined. Three patients were confused and discouraged by their failure. One patient described the experience as follows:

My family told me to cook. I made a dish, but I forget to make another one. "What about the other dish?" my family asked me. I forgot it. I am a no-good person (Participant 8).

\section{Concerns about impacts of cognitive impairment on treatment of breast cancer}

One patient was worried that she would not be properly treated due to cognitive impairment. The patient worried about the recurrence of her breast cancer due to missing medication due to her cognitive impairment:

My husband told me it was okay because forgetting to take the medicine isn't life-threatening, but I'm worried (Participant 3).

\section{Concerns about care burden of their family}

Four patients were also concerned about the burden on their families as their cognitive impairment progressed. One patient described her experience as follows:

I have children, so I can talk about anything, but they have their lives. I don't want to bother my children (Participant 9).

\section{Concerns about progressing cognitive impairments}

Eight patients were concerned about the dementia that was related to their hormone therapy, comorbidities and treatments for them, and aging. One patient thought that their cognitive impairment was caused by the hormone therapy and seven patients expressed concern about the impact of medications for their comorbidities and aging on cognitive function:

I often attributed my cognitive decline to hormone therapy... I have to take Femara ${ }^{\circledR}$ for another 4 years. (Participant 8).

In addition, patients described that they worried about developing dementia. 
Cognitive decline may be due to age, but I think it's because I take a lot of medicine... Other illnesses have occurred and the number of medicines have increased (Participant 8 ).

\section{Feeling secure that their cognitive impairment is not unusual}

Seven patients perceived that cognitive decline was a normal part of aging. Three patients did not report difficulties in their daily life. Seven patients were relieved to know that their situation was similar to their friends. Since the impact of cognitive impairment on daily life was small, it was not regarded as something special. One patient described the experience as follows:

My friend told me that we were all the same. I was relieved to hear from my friends that people lost cognitive function as they got older (Participant 4).

\section{Theme 3: Coping with cognitive impairments}

\section{Coping with problems related to cognitive impairment}

Ten patients had several coping strategies to maintain their daily life. Patients used problem-solving strategies such as being persistent, limiting failure, and seeking support from their family and physician. One patient read documents repeatedly. Seven patients devised a medication management method and confirmed their schedule with notes or a calendar. A patient who concentrated on one thing said:

I used to make two items together, but now I make one dish at a time... When I used to cook more than one dish, I forgot the other one and sometimes it was burnt. Oh, it smelt. Now, I make one and set it aside, and make one and set it aside (Participant 8).

Six patients received support from their family and physicians. In addition, $t$ wo patients used an emotion regulation strategy of not worrying. Two patients also tried not to be distressed or obsessive. Five patients thought positively. One patient described the experience as follows:

I sometimes forget, but the physician said that there was no problem. So, I think it doesn't matter (Participant 6).

\section{Trying to maintain and improve cognitive function}

Four patients worked to enhance cognitive function. Three patients practiced stimulating their brain, such as by reading books, to enhance cognitive function. Two patients actively engaged with people and continued social activities.

I don't talk when I'm alone. I don't have a chance to talk. When I go out, I talk to someone. Whether it's an exercise class or swimming class, I don't talk for a long time, but I talk (Participant 2).

In addition, seven patients actively worked on new activities. 
I started using a personal computer. I was invited to do so by a friend, so I thought I should give it a try (Participant 6).

\section{Discussion}

This study described the experience of cognitive impairment in older patients who received hormone therapy for breast cancer in Japan. The results of the current study had similarities and differences to previous studies $[19,20]$, which explored patients' experience of chemotherapy-related cognitive impairment for breast cancer.

Older people have many risk factors of cognitive impairment. Previous research revealed an association between polypharmacy and cognitive impairment in the elderly in an urban community in Japan [21]. Eight participants were taking multiple medications, and the study participants described the effects of the medications on their cognitive function. On the other hands, some study participants responded that they felt that cognitive decline was not unusual because they were elderly and others recognized that their cognitive function was superior to that of their peers. The range of MMSE scores of the study participants was 28-30, and they did not have difficulties in daily life. Therefore, these study participants may not have considered their current cognitive impairment to be noteworthy. Medical professionals should treat older breast cancer patients' concerns of cognitive impairment by considering the number of oral medications, aging, and patients' perceptions about their cognitive impairment.

Study participants reported cognitive changes in various domains that included memory, language and executive function, concentration, understanding, and clarity. This finding is consistent with a previous study [22]. Therefore, older women receiving hormone therapy for breast cancer should be assess their cognitive function. The MMSE is commonly used to screen for dementia, but its sensitivity for cancer treatment-related cognitive impairment is low [23]. In general, cancer therapy-related cognitive impairment is subtle [24]. Therefore, it is necessary to use a tool for evaluating the cognitive dysfunction related to cancer treatment, such as FACT-Cog [25], to assess their self-reported cognitive function.

Cognitive impairment has economic impact, psychosocial impact, and minimal impact on patients' daily lives [20]. Participants in this study did not talk about the economic impact of their treatment. The impact on economic status may have been small because the study participants were older and none of them worked full-time. Some study participants were psychologically upset by perception of their cognitive impairment. Depressive symptoms affect cognitive function [26, 27]. One patient in this study, who had low MHI-17 depression scores, felt anxiety because her husband, who was receiving treatment for cancer, was hospitalized for fever and his status was not stable. Therefore, it is necessary to assess the mental health of the older patient by considering the health condition of not only the patient but also their family. In addition, some study participants were less motivated to engage in social activities due to their perception of cognitive impairment, and went out less often. Medical professionals need to evaluate impact of cognitive decline on social activities of older people. 
Participants in this study were independent in activities of daily living, but were worried that cognitive dysfunction would put a burden on their families. In a national survey of adult Japanese people, $73.5 \%$ of the respondents reported that one the factors that made them anxious about getting dementia was being a physical and mental burden to their families [28]. It is necessary to develop a support system for patients' families and strategies to compensate for the decline in cognitive function.

Participants of this study coped with problems caused by their cognitive impairment and tried to maintain and improve their cognitive function. Although no effective treatment for the cognitive dysfunction associated with cancer treatment has been established, the interventions recommended in the guidelines [29] may be effective for patients' cognitive function. It is also important to promote coping with cognitive dysfunction. Patients in this study used problem-focused coping and emotion-focused coping to maintain and improve their cognitive function. In a previous study [30], organizational/environmental strategies, social/behavioral strategies, and self-management strategies were recommended for cancer therapy-related cognitive impairment. Medical professionals need to know effective coping strategies and to provide information of strategies that meet the patient's ability and resources.

\section{Study Limitations}

This study has some limitations. First, most of the study participants were in their 70 s and lived with someone. They had high activities of daily living (ADL) and social function, and good mental health status. It has been suggested that physical activity alleviates age-related decline in cognitive function [31] and psychological aspects are factors in cognitive impairment [1]. Furthermore, the previous study indicated that age was related to cognitive decline in women who received chemotherapy for breast cancer [32]. It is necessary to investigate a wider range of subjects (older patients, low ADL, low social function, poor mental health status, or receiving chemotherapy). Second, cognitive dysfunction has a long-term course; however, this study was a cross-sectional study. A longitudinal study of cognitive function in relatively young women (mean: 51.29 years old, range: 30-66 years old) receiving hormone therapy for breast cancer reported no adverse effects of hormone therapy on cognitive function [33]. Older people may be affected by treatment due to the impacts of aging on cognitive function. Finally, the causal relationship between hormone therapy and cognitive impairment was unclear in this study. Therefore, it is necessary to longitudinally examine the cognitive function of older breast cancer survivors before starting hormone therapy.

\section{Conclusion}

This qualitative study, combined with a comprehensive geriatric assessment, explored older patients' experience of living with the cognitive impairment related to hormone therapy for breast cancer. Three themes were generated: recognizing of cognitive function, impacts on mental health, and coping with cognitive impairments. Medical professionals should assess cognitive function in consideration of the comprehensive evaluation of older patients receiving hormone therapy for breast cancer. 


\section{Declarations}

Acknowledgements

The authors gratefully acknowledge all the women who participated in this study. The authors also appreciate the assistance of Dr. Naoki Kagawa of Kagawa Breast Clinic.

\section{Funding}

This study was supported by a Grant-in-Aid for the 24th Scientific Research from the Japanese Breast Cancer Society.

\section{Conflicts of interest}

Naruto Taira reports personal fees from Phizer, Eizai, Actmed, AstraZeneca, and Kyowa Kirin. Yasuko Tenda, Mika Miyashita, Akimitsu Yamada, Chikako Shimizu, Kanako Nakayama, Naoko Honma, and Masataka Sawaki have no financial relationships to disclose.

\section{Availability of data and material}

The data and material are not available publicly as study participants in the current study were informed at the interview survey that their data would be stored strictly.

\section{Code availability}

$\mathrm{N} / \mathrm{A}$

\section{Authors' Contributions}

All authors contributed to the study conception and design. Material preparation and data collection were performed by Yasuko Tenda. Data analysis was performed by Yasuko Tenda and Mika Miyashita. The first draft of the manuscript was written by Mika Miyashita and Yasuko Tenda, and all authors commented on previous versions of the manuscript. All authors read and approved the final manuscript.

\section{Ethics approval}

This study has been approved by the institutional review boards at Hiroshima University (E-1350).

\section{Consent to participate}

Informed consent was obtained from all the women who participated in this study.

\section{Consent for publication}

Study participants signed informed consent regarding publishing their data and interview content with protecting personal information. 


\section{References}

1. Lange M, Joly F, Vardy J, Ahles T, Dubois M, Tron L, Winocur G, De Ruiter MB, Castel H (2019) Cancerrelated cognitive impairment: an update on state of the art, detection, and management strategies in cancer survivors. Ann Oncol 30(12):1925-1940 doi: 10.1093/annonc/mdz410.

2. Jansen C, Miaskowski C, Dodd M, Dowling G, Kramer J (2005) Potential mechanisms for chemotherapy-induced impairments in cognitive function. Oncol Nurs Forum 32(6):1151-1163

3. Von Ah D, Russell KM, Storniolo AM, Carpenter JS (2009) Cognitive dysfunction and its relationship to quality of life in breast cancer survivors. Oncol Nurs Forum 36(3):326-336

4. Park JH, Jung YS, Jung YM, Bae SH (2019) The role of depression in the relationship between cognitive decline and quality of life among breast cancer patients. Support Care Cancer 27(7):27072714 doi: 10.1007/s00520-018-4546-x.

5. Kubo M, Kumamaru H, Isozumi U, Miyashita M, Nagahashi M, Kadoya T, Kojima Y, Aogi K, Hayashi N, Tamura K, Asaga S, Niikura N, Ogo E, lijima K, Tanakura K, Yoshida M, Miyata H, Yamamoto Y, Imoto S, Jinno H (2020) Annual report of the Japanese Breast Cancer Society registry for 2016. Breast Cancer 27(4):511-518

6. Sawaki M, Yamada A, Kumamaru H, Miyata H, Nakayama K, Shimizu C, Miyashita M, Honma N, Taira N, Saji S (2021) Clinicopathological characteristics, practical treatments, prognosis, and clinical issues of older breast cancer patients in Japan. Breast Cancer 28(1):1-8 doi: 10.1007/s12282-02001188-8.

7. Buwalda B, Schagen SB (2013) Is basic research providing answers if adjuvant anti-estrogen treatment of breast cancer can induce cognitive impairment? Life Sci 93(17): 581-588

8. Inouye SK, Studenski S, Tinetti ME, Kuchel GA (2007) Geriatric syndromes: Clinical, research, and policy implications of a core geriatric concept. J Am Geriatr Soc 55(5):780-791

9. Edelstein A, Pergolizzi D, Alici Y (2017) Cancer-related cognitive impairment in older adults. Curr Opin Support Palliat Care 11(1):60-69. doi: 10.1097/SPC.0000000000000254.

10. Frank JS, Vance DE, Triebel KL, Meneses KM (2015) Cognitive deficits in breast cancer survivors after chemotherapy and hormonal therapy. J Neurosci Nurs 47(6):302-312.

11. Wu LM, Amidi A (2017) Cognitive impairment following hormone therapy: current opinion of research in breast and prostate cancer patients. Curr Opin Support Palliat Care 11(1):38-34

12. Blanquicett $C$, Cohen JB, Flowers $C$, Johnson $T 2^{\text {nd }}$ (2019) The Role of the Comprehensive Geriatric Assessment in the Evaluation of the Older Cancer Patient. Oncology (Williston Park) 33(11):687524

13. Extermann M, Hurria A (2007) Comprehensive geriatric assessment for older patients with cancer. J Clin Oncol 25(14):1824-1831 doi: 10.1200/JC0.2007.10.6559.

14. Folstein MF, Folstein SE, McHugh PR (1975) “Mini-mental state”: A practical method for grading the cognitive state of patients for the clinician. J Psychiatr Res 12(3):189-198

15. Hurria A, Gupta S, Zauderer M, Zuckerman EL, Cohen HJ, Muss H, Rodin M, Panageas KS, Holland JC, Saltz L, Kris MG, Noy A, Gomez J, Jakubowski A, Hudis C, Kornblith AB (2005) Developing a 
cancer-specific geriatric assessment: A feasibility study. Cancer 104:1998-2005

16. Stewart AL. Ware JE Jr (1992) Measuring Functioning and Well-Being: The Medical Outcomes Study Approach. Duke University Press, Durham, North Carolina

17. Sherbourne CD, Stewart AL (1991) The MOS social support survey. Soc Sci Med 32(6):705-714 doi: 10.1016/0277-9536(91)90150-b.

18. Elo S, Kyngäs H (2008) The Qualitative Content Analysis Process. J Adv Nurs 62:107-115

19. Myers JS (2012) Chemotherapy-related cognitive impairment: the breast cancer experience. Oncol Nurs Forum 39(1):E31-40 doi: 10.1188/12.0NF.E31-E40.

20. Bolton G, Isaacs A (2018) Women's experiences of cancer-related cognitive impairment, its impact on daily life and care received for it following treatment for breast cancer. Psychol Health Med 23(10):1261-1274 doi: 10.1080/13548506.2018.1500023.

21. Onoue H, Koyama T, Zamami Y, Hagiya H, Tatebe Y, Mikami N, Shinomiya K, Kitamura Y, Hinotsu S, Sendo T, Ouchi Y, Kano MR (2018) Trends in Polypharmacy in Japan: A Nationwide Retrospective Study. J Am Geriatr Soc 66(12):2267-2273

22. Von Ah D, Habermann B, Carpenter JS, Schneider BL (2013) Impact of perceived cognitive impairment in breast cancer survivors. Eur J Oncol Nurs 17(2):236-241

23. Meyers CA, Wefel JS (2003) The use of the mini-mental state examination to assess cognitive functioning in cancer trials: no ifs, ands, buts, or sensitivity. J Clin Oncol 21(19):3557-3558

24. Vardy J, Wefel JS, Ahles T, Tannock IF, Schagen SB (2008) Cancer and cancer-therapy related cognitive dysfunction: an international perspective from the Venice cognitive workshop. Ann Oncol 19(4):623-629

25. Miyashita M, Tsukamoto N, Hashimoto M, Kajiwara K, Kako J, Okamura H (2020) Validation of the Japanese Version of the Functional Assessment of Cancer Therapy-Cognitive Function Version 3. J Pain Symptom Manage 59(1):139-146 e3. doi: 10.1016/j.jpainsymman.2019.09.027.

26. Bedillion MF, Ansell EB, Thomas GA (2019) Cancer treatment effects on cognition and depression: The moderating role of physical activity. Breast 44:73-80

27. Yang Y, Hendrix CC (2018) Cancer-Related Cognitive Impairment in Breast Cancer Patients: Influences of Psychological Variables. Asia Pac J Oncol Nurs 5(3):296-306 doi: 10.4103/apjon.apjon_16_18.

28. Cabinet Office, Government of Japan (2020) Public opinion survey 2019. https://survey.govonline.go.jp/tokubetu/tindex-r01.html Accessed on 2020 March 24 (in Japanese)

29. Oncology Nursing Society (2020) Symptom interventions and guide lines, cognitive impairment. https://www.ons.org/pep/cognitive-impairment. Accessed on 2020 November 22

30. Von Ah D, Storey S, Jansen CE, Allen DH (2013) Coping strategies and interventions for cognitive changes in patients with cancer. Semin Oncol Nurs 29(4):288-299

31. Liu-Ambrose T, Barha CK, Best JR (2018) Physical activity for brain health in older adults. Appl Physiol Nutr Metab 43(11):1105-1112 doi: 10.1139/apnm-2018-0260 
32. Ahles TA, Saykin AJ, McDonald BC, Li Y, Furstenberg CT, Hanscom BS, Mulrooney TJ, Schwartz GN, Kaufman PA (2010) Longitudinal assessment of cognitive changes associated with adjuvant treatment for breast cancer: impact of age and cognitive reserve. J Clin Oncol 28(29):4434-4440 doi: $10.1200 / J C 0.2009 .27 .0827$.

33. Van Dyk K, Crespi CM, Bower JE, Castellon SA, Petersen L, Ganz PA (2019) The cognitive effects of endocrine therapy in survivors of breast cancer: A prospective longitudinal study up to 6 years after treatment. Cancer 125(5):681-689 https://doi.org/10.1002/cncr.31858

\section{Tables}

Due to technical limitations, table 1,2 is only available as a download in the Supplemental Files section.

\section{Supplementary Files}

This is a list of supplementary files associated with this preprint. Click to download.

- Table1.xlsx

- Table2.xlsx 